frequency $\omega$ and electron-atom collision frequency. By this means excitation, ionization and other transport properties, readily measurable under static fields, may be used to deduce optical frequency counterparts. In this way it is possible to derive expressions for the critical radiation intensity required to cause breakdown in gases exposed to laser light; it turns out to be approximately proportional to $\omega^{2} / \tau$ where $\tau$ is the duration of the laser flash (F. Morgan, L. R. Evans and C. G. Morgan, J. Phys. D., 4, in the press).

Comparison between measurement and prediction is remarkably good when the experimental complexities and theoretical approximations are taken into account, but it has been argued that because the classical oscillation energy of the electron in the laser field is small compared to the photon energy the classical description is inapplicable.

Zernik contends that this reasoning is incorrect and he applies a simple extrapolation of the classical theory in the case of breakdown in helium with some success over a wide pressure range.

The $t s 3$ mutation also affects the response of permissive cells supporting the replication of the virus. Usually when polyoma virus replicates in mouse cells, host DNA synthesis is induced concomitant with the replication of the viral genome and the cells are induced to move. At $32^{\circ} \mathrm{C}$ the $t s 3$ mutant behaves in these respects like wild type virus, but at $39^{\circ} \mathrm{C}$ both the induction of cell movement and cell DNA synthesis by $t s 3$ are impaired. It seems unlikely that the $t s 3$ gene product directly controls all these responses in both transformed hamster cells and lytically infected mouse cells. More likely the $t s 3$ gene may specify a molecule which changes the chemistry of the surface of the infected cell. Dulbecco, Eckhart and Burger have evidence (as yet unpublished), that this is indeed the case, but that is another story about which we shall no doubt shortly be reading.

Apart from inducing cellular DNA synthesis, polyoma virus and SV40 replicating in permissive hosts induce the scission of the cell's genome and as a result pieces of cellular DNA of appropriate size are enclosed in capsids of the virus. The possibility that such pseudovirions, as they are called, might be useful as vehicles for the transduction of cells has been widely and often uncritically discussed. The analogy between pseudovirions and certain transducing phage particles remains, however, and Grady, Axelrod and Trilling (Proc. US Nat. Acad. Sci., 67, 1886; 1970) are among the groups pursuing the possibility of showing transduction of eukaryotic cells. They report that the pseudovirions produced when SV40 replicates in the VERO line of monkey cells contain repeated and unique sequences of monkey DNA in the same proportions as in the total VERO cell genome. This indicates, of course, that particular portions of the genome are not preferentially incorporated into pseudovirions. There is therefore no hope of using pseudovirions as analogues of lambda phage for specialized transduction, that is, the transduction at high frequencies of specific portions of a cell's genome. A putative general transducing potential remains, however, and Grady et al. have been able to show that about 10 per cent of the SV40 pseudovirions taken up by mouse embryo cells enter the nucleus carrying their monkey DNA undegraded. Whether such foreign DNA is replicated and expressed, however, is the crucial question which remains unanswered.

\section{Synthesis of Bacterial Cell Walls}

Functions for the techoic acids of Gram positive bacteria are proposed in three articles in next Wednesday's Nature New Biology $(229,1971)$ by a team in the Microbiological Chemistry Research Laboratory at the University of Newcastle upon Tyne. Teichoic acids are polymers composed either of glycerol phosphate or ribitol phosphate, and the principal chain has substituents on it which vary in different strains and include D-alanine, D-glucose, D-galactose or amino sugars. The Newcastle team (J. Baddiley et al.), who first discovered these polymers, suggested in 1961 (Nature, $191,570)$ that they participate in ionexchange reactions in the outer regions of the bacterial cell; the phosphate of the teichoic acids bestows on the isolated cell walls the ability to bind magnesium ions. Baddiley et al. have now isolated functioning cell wall-cell membrane fragments and cell membrane fragments without any cell wall. These fragments catalyse the synthesis of teichoic acids from UDPglucose and CDP-glycerol and require high concentrations of $\mathbf{M g}^{2+}$ ions. Preincubation of the fragments of wall membrane made the membrane enzymes independent of $\mathrm{Mg}^{2+}$ concentration; the pure membrane fractions required $10 \mathrm{mM}$ $\mathrm{Mg}^{2}+$ for maximal activity. This is a direct demonstration of the function of teichoic acids in binding $\mathbf{M g}^{\mathbf{3}}+$ ions and making them available to the membrane.

Chloramphenicol inhibits the biosynthesis of the teichoic acid of a bacterial cell wall in a cell-free system of fragmented cell membrane from Bacillus subtilis. The chloramphenicol seems to inhibit the synthesis of the teichoic acid directly and is unrelated to protein synthesis. The antibiotic inhibits the transfer of glucose from UDP-glucose to the polymer. The inhibition is specific for glucose and does not occur with other nucleotide sugars. The polymer, whose synthesis is inhibited, is unusual in that the glucose is

\section{AAAS \\ Model Biology}

by our Special Correspondent

THE increasing infiltration of biology by physical scientists and mathematicians was plainly reflected in the contribution of the American Mathematical Society to this year's meeting of the American Association for the Advancement of Science (AAAS) in Chicago. The section was devoted entirely to biology; in particular to two of its most fascinating and intractable problems-control of development and central nervous function.

Dr B. C. Goodwin (University of Sussex) gave a somewhat apologetic talk

in the principal chain, rather than attached to it.

The biosynthesis of several bacterial wall polymers uses lipid intermediates that aid in the transport of sugar residues and related components from intracellular nucleotide precursors through the membrane to the polymer chains in the wall. Such lipid intermediates are used in the biosynthesis of both teichoic acids and peptidoglycan, which is a polymer rontaining $\mathrm{N}$-acetylmuramic acid, $\mathrm{N}$-acetylglucosamine, alanine, lysine, glutamic acid and glycine.

The Newcastle group has further examined the possibility that a single lipid may be common to both pathways, and that therefore the availability of this lipid could play some part in the regulation of cell wall synthesis. The amount of these lipids in bacteria is very small and their direct study is therefore difficult. Consequently, Baddiley and his colleagues looked for an indirect answer. If in a single organism the identical lipid is involved in the synthesis of the two polymers, competition for the limited amount of lipid would probably occur. They therefore examined the relationship between the biosynthesis of peptidoglycan and teichoic acid in cell membrane fragments from Staphylococcus lactis I3. Inhibition of teichoic acid synthesis is achieved by the addition of peptidoglycan precursors. The antibiotics bacitracin and vancomycin inhibit the formation of peptidoglycan and prevent the return of the lipid intermediate to the pool; the antibiotics have no direct effect on teichoic acid biosynthesis. When peptidoglycan precursors and the antibiotic are added together, however, thereby effectively trapping the lipid intermediate, a sharp inhibition of teichoic acid synthesis results. It follows that the same lipid carrier molecules are used for transporting precursors of both peptidoglycan and teichoic acid. 